Document downloaded from:

http://hdl.handle.net/10251/84829

This paper must be cited as:

Fornés Leal, A.; García Pardo, C.; Frasson, M.; Pons Beltrán, V.; Cardona Marcet, N. (2016). Dielectric characterization of healthy and malignant colon tissues in the $0.518 \mathrm{GHz}$ frequency band. Physics in Medicine and Biology. 61(20):7334-7346. doi:10.1088/00319155/61/20/7334.

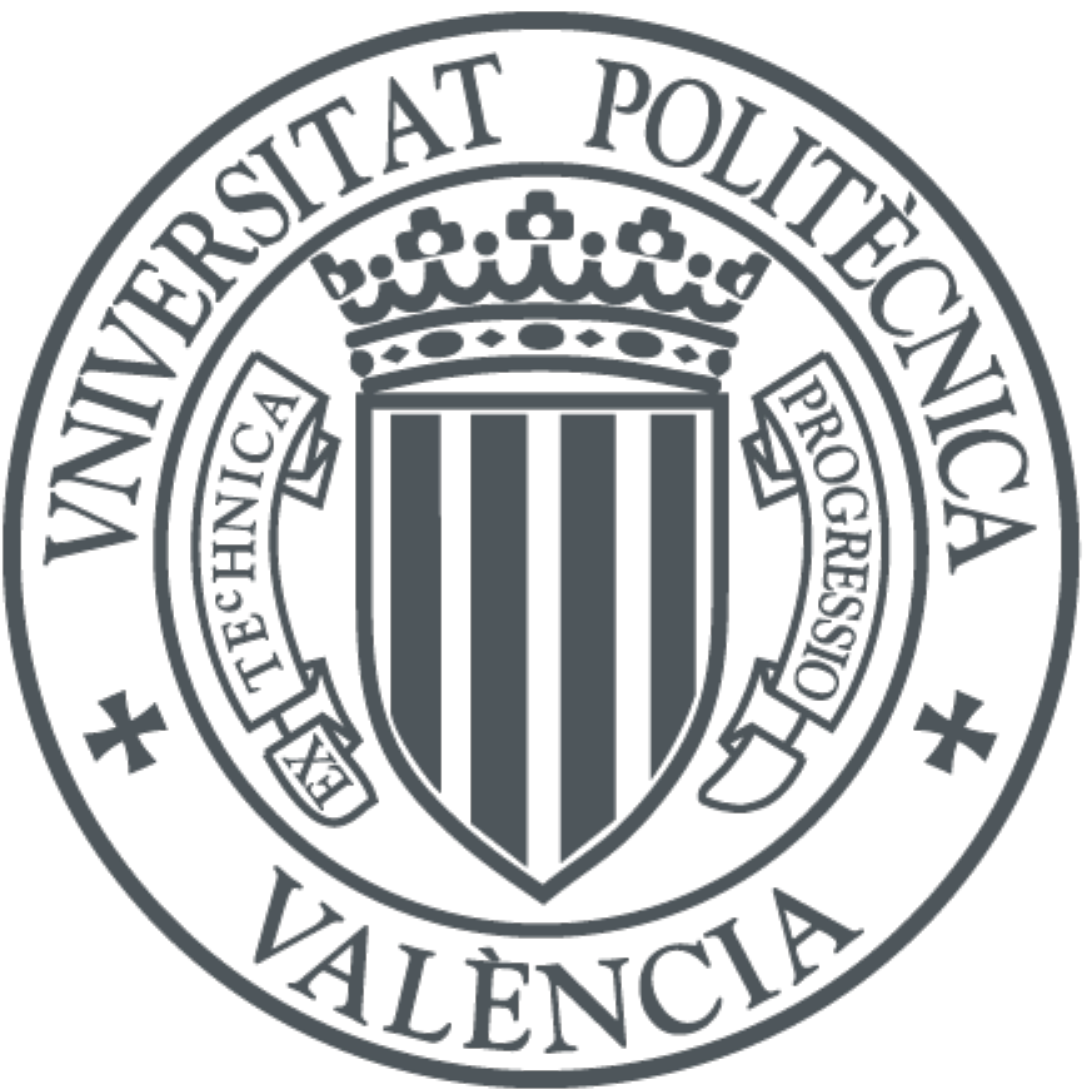

The final publication is available at

http://dx.doi.org/10.1088/0031-9155/61/20/7334

Copyright IOP Publishing

Additional Information 


\title{
Dielectric characterization of healthy and malignant colon tissues in the 0.5-18 GHz frequency band
}

\author{
A Fornes-Leal ${ }^{1}$, C Garcia-Pardo ${ }^{1}$, M Frasson ${ }^{2}$, V Pons Beltrán ${ }^{2}$ and N Cardona ${ }^{1}$ \\ ${ }^{1}$ iTEAM, Universitat Politècnica de València, 46022 València, Spain \\ ${ }^{2}$ Hospital Universitari i Politècnic La Fe, Digestive Diseases Area, 46126 València, Spain \\ E-mail: alforlea@iteam.upv.es
}

\begin{abstract}
Several reports of last decades show that the dielectric properties of healthy and malignant tissues of the same body organ are usually showing different values. However, no large dielectric studies of human colon tissue have been performed, despite being one of the most common types of cancer worldwide. In order to provide information regarding this matter, a dielectric characterization of healthy and malignant colon tissues is presented. Measurements are performed over ex vivo surgery samples obtained from 20 patients, using an open-ended coaxial probe in the $0.5-18 \mathrm{GHz}$ frequency band. Results show that the dielectric constant of colon cancerous tissue is $8.8 \%$ higher than healthy tissues $(p=0.002)$. Besides, conductivity is about $10.6 \%$ higher, but in this case measurements does not have statistical significance $(p=0.038)$. Performing an analysis per patient, the differences in dielectric constant between healthy and malignant tissues appear systematically. Particularized results for specific frequencies $(500 \mathrm{MHz}, 900 \mathrm{MHz}$, $2.45 \mathrm{GHz}, 5 \mathrm{GHz}, 8.5 \mathrm{GHz}$ and $15 \mathrm{GHz}$ ) are also reported. Findings have a potential application in early-stage cancer detection and diagnosis, and can be useful in order to develop new tools for hyperthermia treatments as well as to create electromagnetic models of healthy and cancerous tissues.
\end{abstract}

\section{Introduction}

Cancer has become a worldwide health problem, being the second most common cause of death for all ages combined in the developed countries. In particular, colorectal cancer comprises around the 9.7\% of the global cancer burden in both sexes, and it is the second most common in women and the third in men according to the data presented in (Ferlay et al 2014). The early detection of this kind of cancer is crucial in order to reduce its mortality rate. Thus, screening programs are carried out periodically among the population at risk. The most frequently screening methods used for colorectal cancer include two general categories: stool tests (tests for occult blood or exfoliated DNA) and structural exams (endoscopy and computed tomographic colonography).

Alternatively, microwave techniques are been explored for cancer detection of tissues as well as for treatment procedures. In the past decades, a great number of researchers have given their contribution to the study of the interactions between biological matter and electromagnetic waves. It 
was discovered that malignant and healthy tissues differ in the interaction with such waves because proteins acquire more surface charges in malignant tumours, and the attraction of these charges for water molecules results in the presence of more "bound water" (Bellorofonte et al 2005). Moreover, dramatic changes in metabolism, intercellular communication, and adhesion properties of cancer cells result in the modification of the number and nature of membrane proteins. Thanks to these findings, a prototype of a device containing a nonlinear tuneable oscillator has been developed and used with encouraging results in the diagnosis of prostate (Bellorofonte et al 2005, Da Pozzo et al 2007, Tubaro et al 2008, Gokce et al 2009, Di Viccaro et al 2009), breast (De Cicco et al 2006), thyroid (Sacco et al 2007a), gastric (Sacco et al 2007b), rectum (Vannelli et al 2010) and bladder cancer (Gervino et al 2007). The operating principle of this non-invasive device, known as Bioscanner TRIMprob and presently manufactured by Tema Sinergie (Faenza, Italy), is exposed in (Bellorofonte et al 2005, Vedruccio and Vedruccio 2011). The device is externally approached to the part of the patient's body that is under analysis and can detect a decrement of the received signal at the fundamental frequency if it finds cancerous tissue due to the damping effects of them.

Closely related to the former, it has been proved that the absorption rate in malignant tissues is higher than in their corresponding healthy tissues (Joines et al 1980, Joines 1984). Hence, tissues characterization in terms of their electromagnetic properties (permittivity and conductivity) is crucial to determine their different absorption rates. The knowledge of these properties can be useful for the development of medical applications such as hyperthermia (Siauve and Lormel 2012), as well as an aid in the design of devices for microwave imaging. This technique is based on the contrast in dielectric properties between a cancerous tissue and the normal cells that surrounds it. Thus, previous knowledge of the dielectric properties of the different tissues is crucial for the design of devices. For instance, in (Zhang et al 2013) an antenna conceived to detect malignant breast tissue with this method is tested in a phantom that was developed by using previously reported tissue properties.

Many studies evaluating these properties have been presented. The first works were mostly performed over animal tissues since their electromagnetic properties are similar to their analogous human tissues. Some tissues were measured from 2 to $4 \mathrm{GHz}$ over bovine specimens in (Brady et al 1981). In (Foster and Schepps 1981), healthy and malignant canine tissues were studied to relate their dielectric properties to water content. Gabriel et al (1996a) reviewed the basic concepts of dielectric phenomena in biological tissues and compiled the results of many previous works. In (Gabriel 1996), a huge quantity of healthy tissues (mostly animals) were electromagnetically characterized up to $20 \mathrm{GHz}$. Besides, the dependence of the dielectric properties of tissues with temperature and time was analyzed over bovine liver tissue in (Chin and Sherar 2001).

Lately, many spectroscopy studies have been performed over human tissues. In (Joines et al 1994) several normal and malignant ex vivo human tissue samples were measured in the 50 - 900 $\mathrm{MHz}$ frequency band, showing that the electromagnetic properties of malignant tissues had larger values than those from healthy ones. In (Yoo 2004), similar differences were obtained up to $5 \mathrm{GHz}$. The xenograft model was used, in which human tumours were cultivated in mice, grown, extracted and measured just after the resection. In (O'Rourke et al 2007), authors investigated the differences between normal, malignant and cirrhotic human liver from 0.5 to $20 \mathrm{GHz}$, concluding as well that statistically significant differences exist among the dielectric properties of ex vivo normal and malignant liver tissue. In (Lazebnik et al 2007), a similar study was performed for breast cancer.

Regarding on colon tissue, it has already been addressed in a few reports. In (Joines et al 1994), healthy and malignant human colon tissues were measured, but only up to $900 \mathrm{MHz}$. In (Yoo 2004), normal and malignant colon tissues were cultivated using the xenograft model (explained above) and measured up to $5 \mathrm{GHz}$, without presenting fitting parameters. On the other hand, (Gabriel 1996) is the only report that includes Cole-Cole parameters for this tissue, but measurements were performed over healthy ovine specimens. To the best of our knowledge, there are not studies evaluating both healthy and cancerous colon tissues obtained directly through human excisions up to $18 \mathrm{GHz}$.

Therefore, in this work measurements on human healthy and cancerous colon samples obtained from ex vivo surgery resections are conducted in order to gather information that could assist in the 


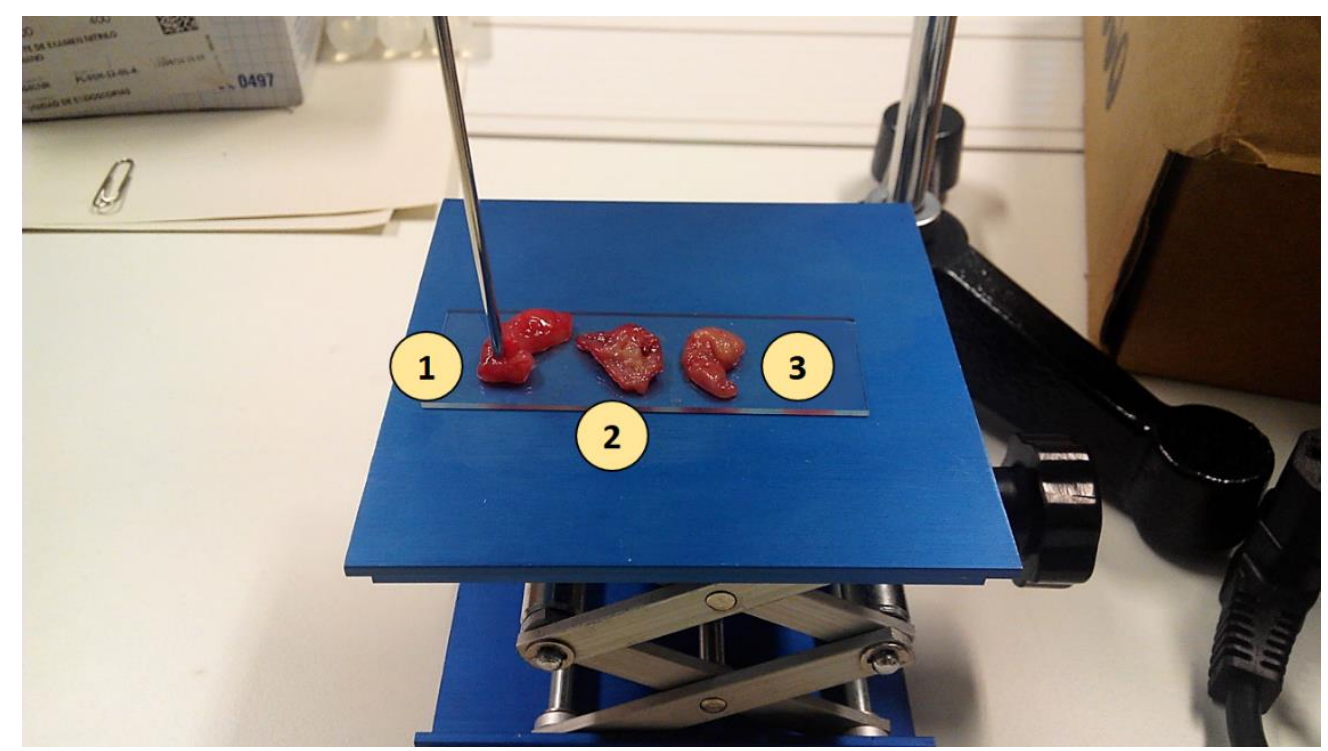

Figure 1. Example of healthy colon wall (1-2) and colon cancer tissue (3).

detection and treatment of colorectal cancer. The dielectric analysis is performed using the openended coaxial technique, which has been used by most of the studies that present electromagnetic properties of biological tissues, like in (Joines et al 1980. Stuchly et al 1982, Joines et al 1994, Gabriel 1996, Chin and Sherar 2001, Yoo 2004, Lazebnik et al 2006, 2007, O'Rourke et al 2007). The working principle of this technique is detailed in (Athey et al 1982, Stuchly et al 1982). This method has been used in different areas, such as chemical mixture (Misra et al 1990), concrete (Filali et al 2008) and moisture characterization (Abbas et al 2005) among others. The dielectric characterization of this study is performed over the $0.5-18 \mathrm{GHz}$ frequency band, particularizing results for six specific frequencies $(500 \mathrm{MHz}, 900 \mathrm{MHz}, 2.45 \mathrm{GHz}, 5 \mathrm{GHz}, 8.5 \mathrm{GHz}$ and $15 \mathrm{GHz}$ ). Results are presented, discussed and compared with previous literature reports.

This paper is organized as follows: in section 2, the procedure of the tissue gathering and the measurement methodology are described. In section 3, the characterization of healthy and malignant colon tissues is presented, and a comparison with previous works is addressed. Finally, conclusions are drawn in section 4.

\section{Methods}

\subsection{Tissue gathering}

The tissue collection protocol was prepared by the project medical supervisors and approved by the ethical committee of the Hospital Universitari i Politècnic La Fe, which is the institution where measurements were driven. Prior to the tissue acquisition, patients were informed of the aim of this clinical study and asked to sign a consent form in which they accepted to take part in it.

The samples used in this work were taken from surgery resections. After surgeons removed the entire cancerous piece from patients, one malignant sample was taken from each excised piece. Since the healthy tissue that surrounds cancer was also resected during surgical procedures, a normal sample was also taken from the excised piece, having thus a healthy (colon mucosa) and a malignant sample of each patient. Samples were placed in different empty plastic receptacles, closed hermetically and sent to the measurement room. The elapsed time between cancer removal and its measurement was approximately 20 minutes. The size of the samples was not constant, as one can observe in figure 1. Still, their width and thickness were at least $1.5 \mathrm{~cm}$ and $0.5 \mathrm{~cm}$ respectively in order to provide rigorous results by avoiding thickness effect, as explained in (Folger $\varnothing$ and Tjomsland 1996, Hagl et al 2003). In this work, healthy and malignant colon tissues of 20 patients have been gathered and characterized. 


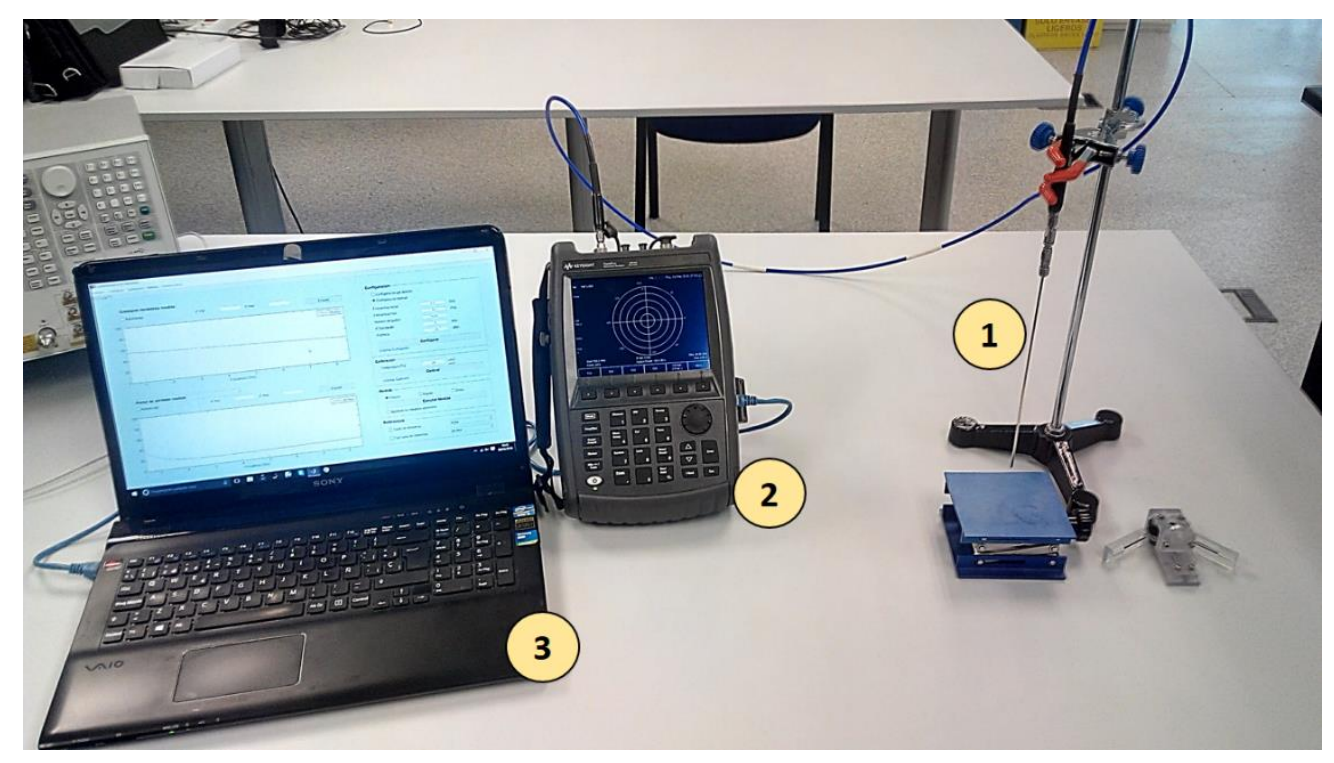

Figure 2. Measurement system used, comprising a commercial open-ended coaxial probe (1), a vector network analyzer (2) and a computer (3), as well as other interconnection instruments (auxiliary coaxial cable, connectors, Ethernet cable) and measurement tools (sample elevator, laboratory stand).

\subsection{Measurement system and data acquisition}

Measurements were conducted in a conditioned room placed in the digestive endoscopy area of Hospital Universitari i Politècnic La Fe of València, Spain. The open-ended coaxial method was used to find the electromagnetic parameters of healthy and malignant tissues. This dielectric characterization system consists of an open-ended coaxial probe (Keysight 85070E Slim Form Probe, $2.2 \mathrm{~mm}$ outer diameter) connected to a vector network analyzer (Keysight Fieldfox N9918A) and controlled by an external computer, as one can see in figure 2 . The vector network analyzer was configured to measure the reflection coefficient at the end of the coaxial probe in the $0.5-18 \mathrm{GHz}$ frequency band with an output power of $-3 \mathrm{dBm}, 1601$ resolution points and $1 \mathrm{kHz}$ of IF bandwidth.

Prior to the arrival of a tissue sample to the measurement room, a calibration of the system was performed, following the guidelines presented in (Marsland and Evans 1987). In this procedure, the reflection coefficients of open circuit (air), short circuit and distilled water were captured and processed in order to relate a reflection coefficient's measurement with its corresponding dielectric parameters. The calibration was checked by measuring a standard $(0.1 \mathrm{M} \mathrm{NaCl}$ solution) and comparing the measured parameters with literature (Buchner et al 1999). Afterwards, the tissue sample was placed on an acrylic sheet over the sample elevator, pressing enough the centre of the tissue with the open-ended coaxial probe to ensure the absence of air between them. After that, its reflection coefficient was captured by the vector network analyzer and its complex relative permittivity $\varepsilon_{\mathrm{r}}$, which is the ratio between the complex permittivity of the tested material and the free space permittivity, was found and stored with the aid of an application controlled by the external computer.

One sample of healthy colon tissue and one sample of malignant colon tissue were measured for each one of the 20 patients. Every sample was measured five times, taking the averaged value.

\subsection{Data analysis}

The dielectric constant $\varepsilon_{\mathrm{r}}$ ' and the conductivity $\sigma$ are the electromagnetic properties of the healthy and malignant colon tissues that are analyzed in the results section. Both electromagnetic properties can be obtained through the measurement of the complex relative permittivity. The dielectric constant, which is the real part of the relative permittivity, is a frequency-dependent property that 
represents the energy that a material stores when an electric field is applied. The conductivity, which is deduced from the imaginary part of the permittivity $\varepsilon_{\mathrm{r}}$ ", comprises the mechanisms of electromagnetic losses inside the material due to electron movement (electric conductivity) and molecular polarization. Conductivity can be computed as:

$$
\sigma(f)=2 \pi f \varepsilon_{r}^{\prime \prime}(f) \varepsilon_{0}
$$

where $\varepsilon_{0}$ is the permittivity of free space $\left(8.854 \times 10^{-12} \mathrm{~F} / \mathrm{m}\right)$. The measurements and the subsequent analysis (mean, standard deviation and distribution fitting) are performed in the $0.5-18 \mathrm{GHz}$ frequency band. Moreover, as commented above, results are also particularized for six frequencies: $500 \mathrm{MHz}, 900 \mathrm{MHz}, 2.45 \mathrm{GHz}, 5 \mathrm{GHz}, 8.5 \mathrm{GHz}$ and $15 \mathrm{GHz}$. These frequencies have been chosen in order to give specific values of our measurements and analyse them in the following sections. Besides, $500 \mathrm{MHz}, 900 \mathrm{MHz}$ and $5 \mathrm{GHz}$ bands allow us to compare our results with literature data. In addition, particularized data for $900 \mathrm{MHz}$ and $2.45 \mathrm{GHz}$ can be useful for the design of devices that work in the $915 \mathrm{MHz}$ and $2.45 \mathrm{GHz}$ ISM bands (electromagnetic properties of 900 and 915 $\mathrm{MHz}$ are almost identical). The last two frequencies ( 8.5 and $15 \mathrm{GHz}$ ) have been chosen for offering specific data at upper frequencies, since there are no more ISM bands licensed until $24.125 \mathrm{GHz}$. However, data for MICS band (403.5 MHz) and 433.92 ISM band have not been provided since our coaxial probe only allows measurements above $500 \mathrm{MHz}$.

The measurements of the statistical significance (P-values) of the differences in the dielectric parameters between healthy and malignant tissues have been computed using the parametric MannWhitney U test for two samples, when necessary.

\section{Results and discussion}

\subsection{Statistical analysis}

In this section, we provide the statistical results obtained for the measured healthy and malignant tissues from the 20 patients. In figure 3, the mean of the dielectric constant $\varepsilon_{\mathrm{r}}$ ' and the conductivity $\sigma$ of all healthy and malignant colon tissues are presented, along with their standard deviation (in error bar format). Comparing both kind of tissues, in figure 3(a) one can observe that the dielectric constant of malignant colon tissue is around five points higher than healthy tissue in absolute terms ( $5.06 \pm 2.49$ averaging up to $4 \mathrm{GHz}$ ), while above $4 \mathrm{GHz}$ this difference tends to decrease. Analyzing the entire frequency band in relative terms, the mean of the dielectric constant of malignant tissues is $8.8 \%$ higher at all frequencies $(p=0.002)$. In (Schepps and Foster 1980) is explained that this significant difference is due to the higher water content that cancerous tissues have. Moreover, since proteins acquire more surface charges in cancerous tissues, water molecules are attracted to them causing an increment of "bound water" (Bellorofonte et al 2005). On the other hand, error bars show a lower standard deviation of the dielectric constant of malignant tissues with respect to the healthy ones in the whole frequency band, showing thus a fewer dispersion of the measurements of this kind of tissues. This effect was also noticed at the liver ex vivo report of (O'Rourke et al 2007).

Regarding the conductivity, in figure 3(b) we observe that the differences between normal and cancerous cells are small and only noticeable above $6 \mathrm{GHz}(p=0.038)$, but they have low statistical significance since error bars are highly superimposed in almost all the studied frequency band. These differences increase with frequency due to the frequency factor that multiplies the imaginary part of the permittivity as one can see in (1). Again, the standard deviation of malignant tissues is lower than healthy ones, as one can observe in the error bars of the figure.

A Kolmogorov-Smirnov test that has been applied over several distribution fitting functions has shown that the measurements of both dielectric constant and conductivity follow a logistic distribution around the mean at most frequencies. The logistic is a kind of continuous distribution that is similar in shape to the normal or Gaussian distribution but with heavier tails. 


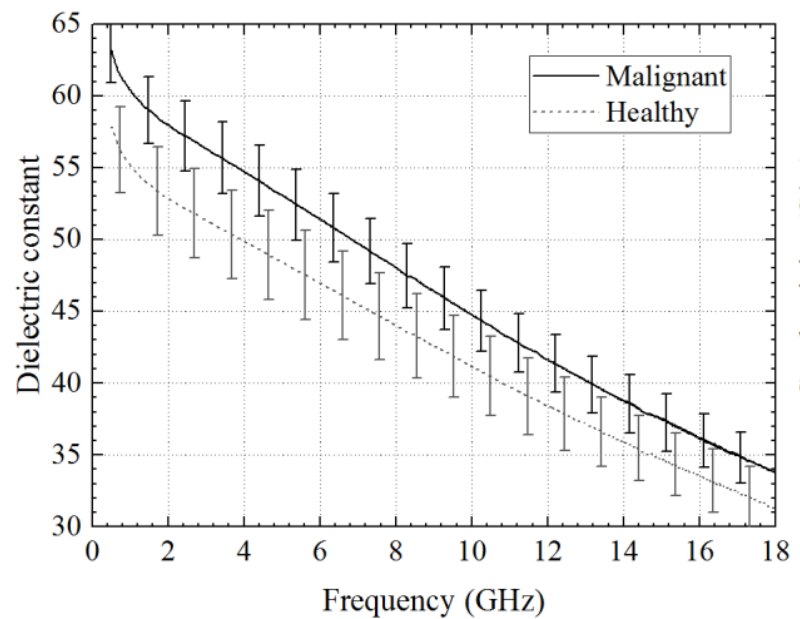

(a)

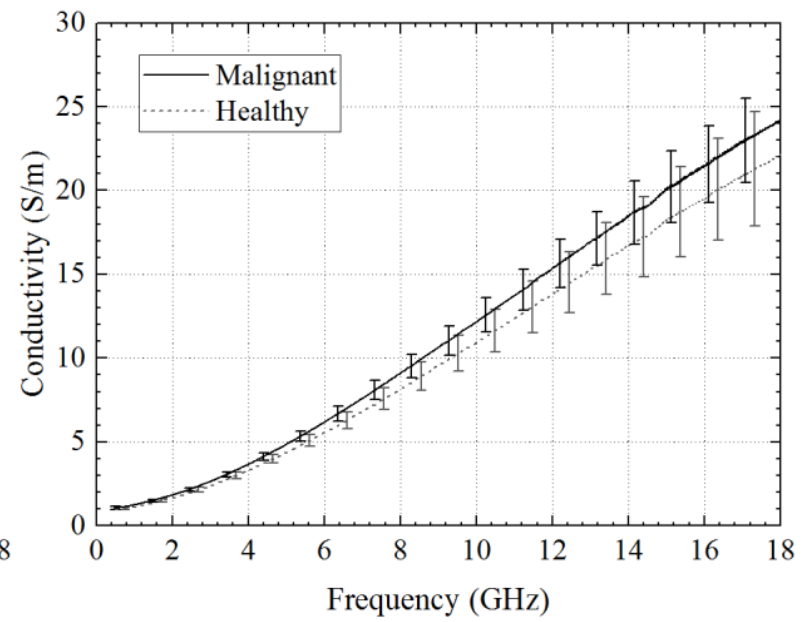

(b)

Figure 3. Mean of the dielectric constant (a) and the conductivity (b) of healthy and malignant colon tissue, along with their standard deviation in error bar format.

The mean and the standard deviation of the dielectric parameters of healthy and malignant tissue for the six discrete frequencies are presented in table 1. One can observe that the absolute differences between normal and malignant tissues are more noticeable in dielectric constant rather than in conductivity. However, in percentage terms the differences are similar. The relative difference in dielectric constant between both types of tissues is $8.9 \%(p<0.001), 9.5 \%(p<0.001), 9.7 \%(p<$ $0.001), 9.6 \%(p<0.001), 9.0 \%(p<0.001)$ and $8.0 \%(p<0.001)$ from the lowest to the upper discrete frequency respectively, whereas the relative difference in conductivity is $9.5 \%(p=0.003)$, $8.9 \%(p=0.001), 9.7 \%(p<0.001), 11.2 \%(p<0.001), 11.6 \%(p<0.001)$ and $10.2 \%(p=0.011)$, respectively.

Table 1. Statistical data of healthy and malignant measurements. Mean and standard deviation values.

\begin{tabular}{ccccc}
\hline \multirow{2}{*}{ Frequency } & \multicolumn{2}{c}{ Malignant Tissues } & \multicolumn{2}{c}{ Healthy Tissues } \\
\cline { 2 - 5 } & $\varepsilon_{\mathrm{r}}{ }^{\prime}$ & $\sigma(\mathrm{S} / \mathrm{m})$ & $\varepsilon_{\mathrm{r}}{ }^{\prime}$ & $\sigma(\mathrm{S} / \mathrm{m})$ \\
\hline $500 \mathrm{MHz}$ & $63.16 \pm 2.05$ & $1.06 \pm 0.08$ & $57.86 \pm 2.96$ & $0.97 \pm 0.08$ \\
$900 \mathrm{MHz}$ & $60.80 \pm 2.27$ & $1.20 \pm 0.09$ & $55.55 \pm 3.06$ & $1.10 \pm 0.09$ \\
$2.45 \mathrm{GHz}$ & $57.19 \pm 2.46$ & $2.17 \pm 0.10$ & $52.14 \pm 3.11$ & $1.98 \pm 0.12$ \\
$5 \mathrm{GHz}$ & $53.10 \pm 2.49$ & $4.87 \pm 0.28$ & $48.46 \pm 3.12$ & $4.38 \pm 0.30$ \\
$8.5 \mathrm{GHz}$ & $47.25 \pm 2.22$ & $9.89 \pm 0.74$ & $43.35 \pm 2.94$ & $8.86 \pm 0.84$ \\
$15 \mathrm{GHz}$ & $37.47 \pm 2.02$ & $20.11 \pm 2.21$ & $34.68 \pm 2.33$ & $18.24 \pm 2.68$ \\
\hline
\end{tabular}

The complex permittivity of biological tissues exhibits several dispersion regions in which its value varies strongly with frequency (Vander Vorst et al 2006). At these regions, the imaginary part of the relative permittivity (known as loss factor) shows a local maximum, whereas the dielectric constant has steeper slopes. The most significant dispersion regions are the $\alpha, \beta, \delta$ and $\gamma$, which affect at different frequency bands (Martinsen et al 2002, Wolf et al 2012). In order to make the permittivity data of biological tissues accessible, results are usually fitted to a Cole-Cole equation of different poles (Gabriel et al 1996b, Lazebnik et al 2006), with each pole characterizing a specific dispersion region. We fit our results using two poles of the Cole-Cole equation since only two dispersion regions ( $\delta$ and $\gamma$ ) can be appreciated in figure 3(a): 


$$
\varepsilon(f)=\varepsilon_{\infty}+\sum_{m=1}^{M} \frac{\Delta \varepsilon_{m}}{1+\left(j \omega \tau_{m}\right)^{\left(1-\alpha_{m}\right)}}+\frac{\sigma_{s}}{j \omega \varepsilon_{0}}
$$

where $M$ is the number of poles of the equation, $\omega$ is the angular frequency $(2 \pi f), \varepsilon_{0}(\mathrm{~F} / \mathrm{m})$ is the permittivity of free space and $\varepsilon_{\propto}, \Delta \varepsilon_{m}, \tau_{m}(\mathrm{~s}), \alpha_{m}$ and $\sigma_{s}(\mathrm{~S} / \mathrm{m})$ are the fitting coefficients of (2). These coefficients are computed in MATLAB for the mean of both normal and malignant data minimizing the sum of squares and using the trust-region-reflective method, which is based on trust regions.

Table 2. Fitting coefficients of (2) for our healthy and malignant measurements.

\begin{tabular}{c|cccccccc}
\hline \multirow{2}{*}{ Malignant } & $\varepsilon_{\propto}$ & $\Delta \varepsilon_{1}$ & $\tau_{1}(\mathrm{ps})$ & $\alpha_{1}$ & $\Delta \varepsilon_{2}$ & $\tau_{2}(\mathrm{~ns})$ & $\alpha_{2}$ & $\sigma_{s}(\mathrm{~S} / \mathrm{m})$ \\
& 3.690 & 55.268 & 7.915 & 0.114 & $0.983 \mathrm{e} 3$ & 10.900 & $6.490 \mathrm{e}-2$ & 0.005 \\
\hline \multirow{2}{*}{ Healthy } & $\varepsilon_{\propto}$ & $\Delta \varepsilon_{1}$ & $\tau_{1}(\mathrm{ps})$ & $\alpha_{1}$ & $\Delta \varepsilon_{2}$ & $\tau_{2}(\mathrm{~ns})$ & $\alpha_{2}$ & $\sigma_{s}(\mathrm{~S} / \mathrm{m})$ \\
& 2.746 & 50.580 & 7.487 & 0.116 & $1.196 \mathrm{e} 3$ & 16.090 & $8.530 \mathrm{e}-2$ & 0.005 \\
\hline
\end{tabular}

The first pole is the most significant of the Cole-Cole fitting curve for the studied frequency band. This pole describes the $\gamma$-dispersion, which is related to the relaxation frequency of water (around 20 $\mathrm{GHz}$ at room temperature) and thus with the water content of tissues. One can observe that the relaxation time $\left(\tau_{1}\right)$ of this pole is slightly higher for the malignant fitting, which implies a lower relaxation frequency. Nevertheless, the value of this parameter is similar in both fittings, which indicates similar trends of the permittivity curves. The term $\Delta \varepsilon_{1}$ is also higher for the malignant fitting, being a term related to the static dielectric constant (dielectric constant when frequency tends to zero). This parameter implies higher values of dielectric constant at the frequencies under study since the trend of the healthy and the malignant curves are almost the same, as mentioned before.

On the other hand, the second pole characterizes the previous dispersion region ( $\delta$-dispersion), and it is computed in order to take into account the change of trend in dielectric constant that can be noticed at frequencies below $1 \mathrm{GHz}$ in figure 3(a). This dispersion is usually ascribed to the relaxation of bound water molecules, but other mechanisms like intra-protein motions seem to affect at those frequencies (Foster and Schwan 1989, Vander Vorst et al 2006, Wolf et al 2012). The fitting values of this dispersion are not very meaningful since part of this relaxation ( $f<500 \mathrm{MHz}$ ) has not been measured.

The parametric values found for our healthy measurements are in agreement with those presented by (Gabriel 1996), especially those of the $\gamma$-dispersion (Gabriel (1996) data of the first pole: $\varepsilon_{\propto}=4$, $\left.\Delta \varepsilon_{1}=50, \tau_{1}=7.958 \mathrm{ps}, \alpha_{1}=0.2\right)$. To the best of our knowledge, it is the only report that includes Cole-Cole parameters for colon tissue. The differences in $\varepsilon_{\propto}, \Delta \varepsilon_{1}$ and $\tau_{1}$ between healthy and malignant tissues are in the same order than those presented in (O'Rourke et al 2007), in this last case from human ex vivo liver tissue.

\subsection{Difference between healthy and malignant tissues among patients}

In this section, we compute the difference in dielectric constant between the healthy and the malignant tissue of each patient $\left(\Delta \varepsilon_{\mathrm{r}}{ }^{\prime}\right)$, and then we analyse how this difference varies among the 20 patients (in terms of its probability distribution and standard deviation). Although the mean of this difference can be computed almost directly from the wideband data, its standard deviation should be studied in order to evaluate the dispersion of such difference in every patient. A KolmogorovSmirnov test has been performed at each frequency testing several distribution functions in order to characterize the kind of distribution that better fits the difference between tissues of the same patient. The test has shown that the logistic distribution is the one that better fits this difference at most frequencies. For instance, the difference at $2.45 \mathrm{GHz}$ follows a logistic distribution of mean $\mu=$ 5.04 and a scale parameter $s=1.37$, as shown in figure 4(a). 


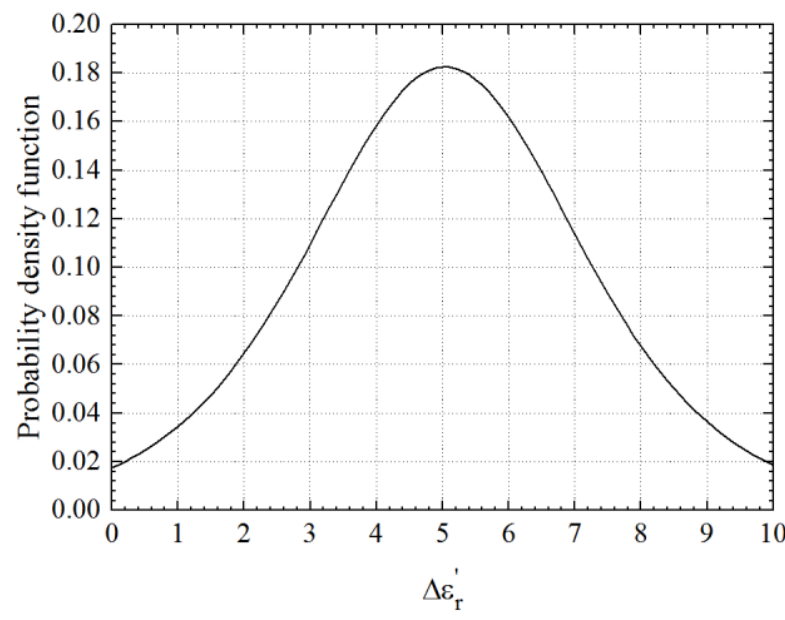

(a)

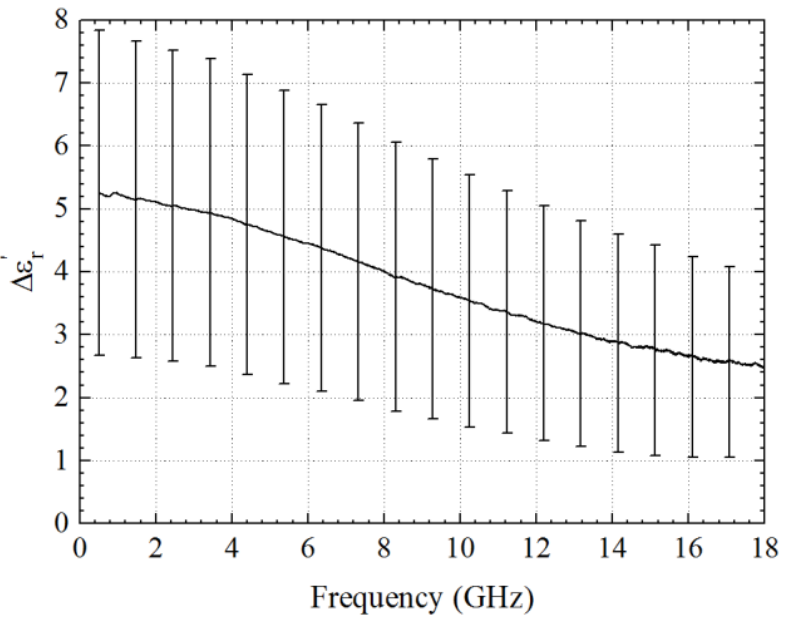

(b)

Figure 4. Statistics of the difference in dielectric constant between the healthy and the malignant tissue from the same patient: (a) probability distribution at $2.45 \mathrm{GHz}$ and (b) mean difference along with its standard deviation in error bar format.

In figure 4(b), the averaged difference in dielectric constant between the healthy and the malignant tissues of the same patient is presented, as well as its standard deviation (in error bar format). This difference is statistically significant at the studied frequency band $(p=0.002)$, and its value is higher at lower frequencies ( $\mathrm{f}<3 \mathrm{GHz}$ ). This difference is reduced gradually with the increment of frequency, also in relative terms. This decrement could be explained by the information presented in (Joines et al 1980, Joines 1984). In these works is exposed that the greatest difference between the electromagnetic absorption of healthy and malignant tissues has a maximum peak between 100 and $800 \mathrm{MHz}$, centred approximately in $400 \mathrm{MHz}$, which can be related to the maximum differences of permittivity among them. Data particularized for the discrete frequencies are presented in table 3 .

Table 3. Averaged difference in dielectric constant $\Delta \varepsilon_{\mathrm{r}}$ ' and conductivity $\Delta \sigma$ between the healthy and the malignant tissue from the same patient. Mean and standard deviation values.

\begin{tabular}{ccc}
\hline Frequency & $\Delta \varepsilon_{\mathrm{r}}{ }^{\prime}$ & $\Delta \sigma(\mathrm{S} / \mathrm{m})$ \\
\hline $500 \mathrm{MHz}$ & $5.25 \pm 2.58^{\mathrm{a}}$ & $9.10 \mathrm{e}-2 \pm 8.40 \mathrm{e}-2$ \\
$900 \mathrm{MHz}$ & $5.25 \pm 2.57$ & $9.86 \mathrm{e}-2 \pm 8.72 \mathrm{e}-2$ \\
$2.45 \mathrm{GHz}$ & $5.04 \pm 2.61$ & $0.19 \pm 0.12$ \\
$5 \mathrm{GHz}$ & $4.63 \pm 2.54$ & $0.49 \pm 0.29$ \\
$8.5 \mathrm{GHz}$ & $3.90 \pm 2.42$ & $1.03 \pm 0.65$ \\
$15 \mathrm{GHz}$ & $2.79 \pm 2.13$ & $1.86 \pm 1.55$ \\
\hline
\end{tabular}

${ }^{\mathrm{a}}$ Standard deviation of the measurement.

The mean differences in conductivity $\Delta \sigma$ are very low and thus have a low practical significance, especially at low frequencies as one can see in table $3(p=0.038)$. On the contrary, differences in dielectric constant $\Delta \varepsilon_{\mathrm{r}}$ ' are high as well as statistically significant $(p=0.002)$, being more noticeable at lower frequencies as commented above. 


\subsection{Comparison with previous studies}

In order to validate our results, the dielectric properties of healthy and malignant colon tissues that have been obtained in this work are next compared to previously published data in which colon tissue dielectric spectroscopy is also addressed. The gathered information from literature is listed in table 4 and represented in figure 5.

Table 4. Previously published works on ex vivo dielectric information of colon tissue.

\begin{tabular}{ccccc}
\hline Source & Type & Frequency & Fitted Data $^{\mathrm{a}}$ & Reference \\
\hline Ovine & Healthy & $10 \mathrm{~Hz}-20 \mathrm{GHz}$ & Yes & (Gabriel 1996) \\
Human & Healthy, Malignant & $50 \mathrm{MHz}-900 \mathrm{MHz}$ & No & (Joines et al 1994) \\
Human $^{\mathrm{b}}$ & Malignant & $200 \mathrm{MHz}-5 \mathrm{GHz}$ & No & (Yoo 2004) \\
\hline
\end{tabular}

${ }^{\mathrm{a}}$ A fitting equation of the results is available.

${ }^{\mathrm{b}}$ Malignant human colon tissue cultivated in mice.

Regarding healthy colon tissue, (Gabriel 1996) is the only work that presents dielectric measurements along with the coefficients of a fitting equation. Although the measurements of this source were performed over ovine tissue, this is the most important and spread study of biological dielectric spectroscopy. In figure 5, one can observe that both the dielectric constant and the conductivity reported in (Gabriel 1996) are very similar to those obtained through our healthy measurements, especially in conductivity (almost identical in the whole frequency band). Regarding the dielectric constant from (Gabriel 1996), although it is slightly higher at lower frequencies, it tends to converge to our results as frequency increases. On the contrary, (Joines et al 1994) colon healthy parameters are too distant from our measurements and (Gabriel 1996) values, although the trends are similar.

In table 5, the dielectric parameters of malignant colon tissue obtained in this work are compared to those obtained in previously published studies (only discrete data available). In (Joines et al 1994), ex vivo human colon tissue was measured up to $900 \mathrm{MHz}$. In (Yoo 2004), malignant colon tissue was also measured, in this case up to $5 \mathrm{GHz}$. In this work, human tumours were cultivated in mice, grown, extracted and measured just after the resection using the xenograft method. The comparison is performed in four of the discrete frequencies (when data are available).

Table 5. Dielectric parameters for the three different colon malignant sources. Mean and standard deviation values.

\begin{tabular}{|c|c|c|c|c|c|c|}
\hline \multirow{2}{*}{ Frequency } & \multicolumn{2}{|c|}{ Present study } & \multicolumn{2}{|c|}{$\left(\right.$ (Joines et al 1994) $^{\mathrm{a}}$} & \multicolumn{2}{|c|}{$(\text { Yoo 2004) })^{b}$} \\
\hline & $\varepsilon_{\mathrm{r}}^{\prime}$ & $\sigma(\mathrm{S} / \mathrm{m})$ & $\varepsilon_{\mathrm{r}}^{\prime}$ & $\sigma(\mathrm{S} / \mathrm{m})$ & $\varepsilon_{\mathrm{r}}^{\prime}$ & $\sigma(\mathrm{S} / \mathrm{m})$ \\
\hline $500 \mathrm{MHz}$ & $63.16 \pm 2.05$ & $1.06 \pm 0.08$ & $57.50 \pm 12.07$ & $0.90 \pm 0.24$ & $64.86 \pm 2.46$ & $1.17 \pm 0.04$ \\
\hline $900 \mathrm{MHz}$ & $60.80 \pm 2.27$ & $1.20 \pm 0.09$ & $56.00 \pm 11.76$ & $1.08 \pm 0.29$ & $62.75 \pm 2.38$ & $1.32 \pm 0.05$ \\
\hline $2.45 \mathrm{GHz}$ & $57.19 \pm 2.46$ & $2.17 \pm 0.10$ & - & - & $59.72 \pm 2.27$ & $2.33 \pm 0.09$ \\
\hline $5 \mathrm{GHz}$ & $53.10 \pm 2.49$ & $4.87 \pm 0.28$ & - & - & $55.95 \pm 2.13$ & $5.55 \pm 0.21$ \\
\hline
\end{tabular}

One can observe that both the dielectric constant and the conductivity from (Yoo 2004) are always higher than those obtained in our study at all frequencies, which in turn are always higher than those from (Joines et al 1994). In particular, our malignant measurements are very similar to those from (Yoo 2004). These differences in the absolute values can be explained by the distinct mean elapsed times between the excisions and measurements of each study, as well as the different 


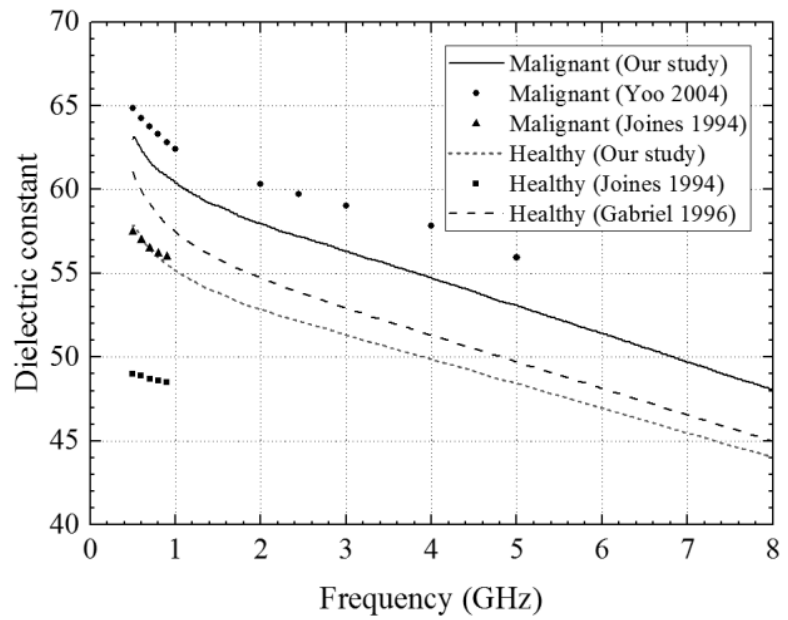

(a)

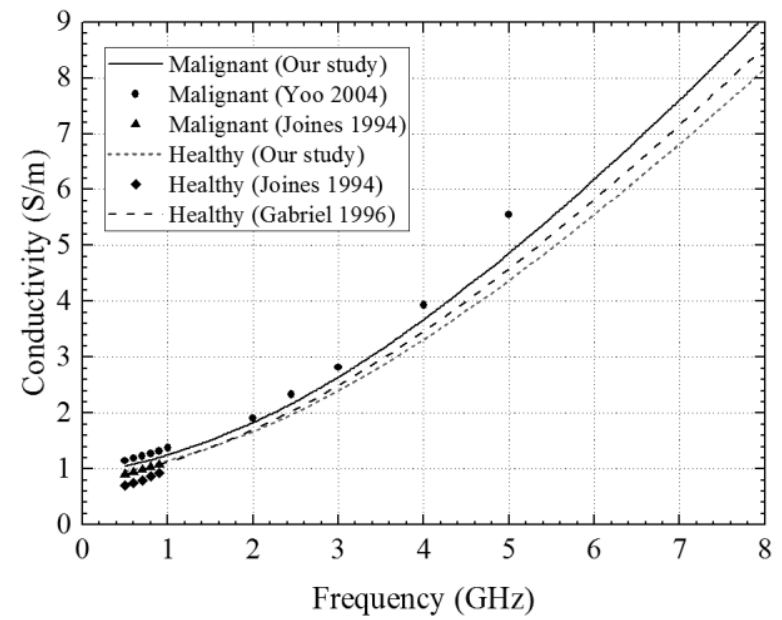

(b)

Figure 5. Dielectric constant (a) and conductivity (b) of healthy and malignant colon tissues obtained in our study and reported in literature.

sample source and preparation. As we commented previously, the elapsed time from excision time to the dielectric characterization is approximately 20 minutes in our work. On the other hand, in (Yoo 2004) this time is sharply reduced because measurements were performed just after the removal of the tumour sample, whereas in (Joines et al 1994) this time is extended between one and two hours. Even though the absolute values of the dielectric parameters of the three sources are different, one can observe similar trends among them with the increment of the frequency. The dielectric constant decreases 2.36, 1.50 and 2.11 points in our study, (Joines et al 1994) and (Yoo 2004) respectively when frequency is increased from $500 \mathrm{MHz}$ to $900 \mathrm{MHz}$. Besides, conductivity rises $0.14,0.18$ and $0.17 \mathrm{~S} / \mathrm{m}$ respectively, almost the same increase in the three sources with the same frequency increment.

Given the big size of the analyzed samples and the short elapsed times between their collection and measurement, the presented ex vivo spectroscopy results should be very similar to measurements obtained through in vivo conditions. However, since some works in which other cancerous tissues have been analyzed report the apparition of unexpected trends or an extra relaxation mechanism around $15 \mathrm{GHz}$ in in vivo conditions (Choi et al 2004, O'Rourke et al 2007), additional measurements should be perform in order to confirm or discard these mechanisms in colon tissue.

\section{Conclusions}

In this study, the electromagnetic properties of ex vivo healthy and malignant colon human tissues have been presented. Tissues were gathered from 20 ex vivo surgery resections. Measurements were performed using the open-ended coaxial technique in the $0.5-18 \mathrm{GHz}$ frequency band, particularizing results as well for six specific frequencies $(500 \mathrm{MHz}, 900 \mathrm{MHz}, 2.45 \mathrm{GHz}, 5 \mathrm{GHz}, 8.5 \mathrm{GHz}$ and 15 $\mathrm{GHz}$ ).

The values obtained for healthy and malignant tissues are in agreement with previously published results, with slightly differences in their values probably caused by the diverse elapsed times between tissue removal and measurement as well as distinct tissue sources and preparation (e.g., xenograft model in mice). For the full band of frequencies analysed, the dielectric constant of malignant colon tissue is on average $8.8 \%$ higher than the dielectric constant of healthy one $(p=$ 0.002). This difference is even higher at frequencies below $4 \mathrm{GHz}$. Regarding the conductivity, the relative difference has a similar behaviour, despite that the absolute differences are much lower than in dielectric constant. The within-patient analysis has shown greater dielectric constants from malignant tissues when compared to healthy tissues, with significant differences that appear systematically. 
These results have a potential application in cancer detection and diagnosis, and can be useful in order to develop new diagnosis devices, tools for hyperthermia treatments and to create electromagnetic models of healthy and malignant colon tissues. Further colon in vivo measurements should be performed in order to confirm the reported trends in this condition, as well as to assess the dielectric behaviour of additional colon lesions.

\section{Acknowledgments}

The authors would like to thank the medical staff of the endoscopy unit of the Hospital Universitari i Politècnic La Fe for their assistance in the gathering of tissue samples. This work was supported by the Ministerio de Economía y Competitividad, Spain (ref. TEC2014-60258-C2-1-R, TEC201456469-REDT), by the European FEDER funds, and by an UPV-IISLaFe action (CEI-2G, 2014).

\section{References}

Abbas Z, You Kok Yeow, Shaari A H, Khalid K, Hassan J and Saion E 2005 Complex permittivity and moisture measurements of oil palm fruits using an open-ended coaxial sensor IEEE Sensors Journal $\mathbf{5}$ 1281-7

Athey T W, Stuchly M A and Stuchly S S 1982 Measurement of radio frequency permittivity of biological tissues with an open-ended coaxial line: Part I IEEE Trans. Microw. Theory Tech. 30 82-6

Bellorofonte C, Vedruccio C, Tombolini P, Ruoppolo M and Tubaro A 2005 Non-invasive detection of prostate cancer by electromagnetic interaction Eur. Urol. 47 29-37

Brady M M, Symons S A and Stuchly S S 1981 Dielectric behavior of selected animal tissues in vitro at frequencies from 2 to $4 \mathrm{GHz}$ IEEE Trans. Biomed. Eng. 28 305-7

Buchner R, Hefter G T and May P M 1999 Dielectric relaxation of aqueous $\mathrm{NaCl}$ solutions J. Phys. Chem. A $1031-9$

Chin L and Sherar M 2001 Changes in dielectric properties of ex vivo bovine liver at $915 \mathrm{MHz}$ during heating Phys. Med. Biol. 46 197-211

Choi J W et al. 2004 Microwave detection of metastasized breast cancer cells in the lymph node; potential application for sentinel lymphadenectomy Breast Cancer Res. Treat. 86 107-15

Da Pozzo L et al 2007 Tissue-resonance interaction method for the noninvasive diagnosis of prostate cancer: analysis of a multicentre clinical evaluation BJU Int. 100 1055-9

De Cicco C et al 2006 Clinical application of spectral electromagnetic interaction in breast cancer: diagnostic results of a pilot study Tumori 92 207-12

Di Viccaro D, Perugia G, Cerulli C, Olivieri V, Bova J, Zanza C, Teodonio S and Liberti M 2009 The accuracy of tissue resonance interaction method probe (Trimprob tm) in non-invasive diagnosis of prostatic cancer. Analysis of the results of 782 patient Urologia 76 Suppl 15 1-3

Ferlay J, Soerjomataram I, Dikshit R, Eser S, Mathers C, Rebelo M, Parkin D M, Forman D and Bray F 2014 Cancer incidence and mortality worldwide: sources, methods and major patterns in GLOBOCAN 2012 Int. J. Cancer 136 E359-86

Filali B, Boone F, Rhazi J and Ballivy G 2008 Design and calibration of a large open-ended coaxial probe for the measurement of the dielectric properties of concrete IEEE Trans. Microw. Theory Tech. $5622322-8$

Folgerø K and Tjomsland T 1996 Permittivity measurement of thin liquid layers using open-ended coaxial probes Meas. Sci. Technol. 7 1164-73

Foster K R and Schepps J L 1981 Dielectric properties of tumor and normal tissues at radio through microwave frequencies J. Microw. Power 16 107-19

Foster K R and Schwan H P 1989 Dielectric properties of tissues and biological materials: a critical review Crit. Rev. Biomed. Eng. 17 25-104

Gabriel C 1996 Compilation of the dielectric properties of body tissues at RF and microwave frequencies Technical Report AL/OE-TR-1996-0037 Armstrong Laboratory, Brooks Air Force Base

Gabriel C, Gabriel S and Corthout E 1996a The dielectric properties of biological tissues: I. Literature survey Phys. Med. Biol. 41 2231-49

Gabriel S, Lau R W and Gabriel C 1996b The dielectric properties of biological tissues: III. Parametric models for the dielectric spectrum of tissues Phys. Med. Biol. 41 2271-93

Gervino G, Autino E, Kolomoets E, Leucci G and Balma M 2007 Diagnosis of bladder cancer at $465 \mathrm{MHz}$ 
Electromagn. Biol. Med. 26 119-34

Gokce O, Sanli O, Salmaslioglu A, Tunaci A, Ozsoy C and Ozcan F 2009 Tissue Resonance Interaction Method (TRIMprob) has the potential to be used alongside the recognized tests in the screening protocols for prostate cancer Int. J. Urol. 16 580-3

Hagl D M, Popovic D, Hagness S C, Booske J H and Okoniewski M 2003 Sensing volume of open-ended coaxial probes for dielectric characterization of breast tissue at microwave frequencies IEEE Trans. Microw. Theory Tech. 51 1194-206

Joines W T 1984 Frequency-dependent absorption of electromagnetic energy in biological tissue IEEE Trans. Biomed. Eng. 31 17-20

Joines W T, Jirtle R L, Rafal M D and Schaefer D J 1980 Microwave power absorption differences between normal and malignant tissue Int. J. Radiat. Oncol. Biol. Phys. 6 681-7

Joines W T, Zhang Y, Li C and Jirtle R L 1994 The measured electrical properties of normal and malignant human tissues from 50 to $900 \mathrm{MHz}$ Med. Phys. 21 547-50

Lazebnik M, Converse M C, Booske J H and Hagness S C 2006 Ultrawideband temperature-dependent dielectric properties of animal liver tissue in the microwave frequency range Phys. Med. Biol. 51 194155

Lazebnik M et al 2007 A large-scale study of the ultrawideband microwave dielectric properties of normal, benign and malignant breast tissues obtained from cancer surgeries Phys. Med. Biol. 52 6093-115

Marsland T P and Evans S 1987 Dielectric measurements with an open-ended coaxial probe IEE Proc. $H$ Microwaves, Antennas and Propagation 134 341-9

Martinsen O G, Grimnes S and Schwan H P 2002 Interface phenomena and dielectric properties of biological tissue Encycl. Surf. Colloid Sci. 2 2643-52

Misra D, Chabbra M, Epstein B R, Microtznik M and Foster K R 1990 Noninvasive electrical characterization of materials at microwave frequencies using an open-ended coaxial line: test of an improved calibration technique IEEE Trans. Microw. Theory Tech. 38 8-14

O'Rourke A P, Lazebnik M, Bertram J M, Converse M C, Hagness S C, Webster J G and Mahvi D M 2007 Dielectric properties of human normal, malignant and cirrhotic liver tissue: in vivo and ex vivo measurements from 0.5 to $20 \mathrm{GHz}$ using a precision open-ended coaxial probe. Phys. Med. Biol. 52 4707-19

Sacco R, Innaro N, Pata F, Lucisano A M, Talarico C and Aversa S 2007a Preoperative diagnosis of incidental carcinoma in multinodular goitre by means of electromagnetic interactions Chir Ital $\mathbf{5 9} 247-51$

Sacco R, Sammarco G, De Vinci R, Vescio G, Scarpelli A, Lucisano A M, Pata F, Mascia E and Martines V 2007b Relief of gastric cancer with an electromagnetic interaction system (TRIMprob) in outpatients Chir. Ital. 59 823-8

Schepps J L and Foster K R 1980 The UHF and microwave dielectric properties of normal and tumour tissues: variation in dielectric properties with tissue water content Phys. Med. Biol. 25 1149-59

Siauve N and Lormel C 2012 Interstitial microwave hyperthermia treatment investigations J. Phys. Conf. Ser. 395012001

Stuchly M A, Athey T W, Samaras G M and Taylor G E 1982 Measurement of radio frequency permittivity of biological tissues with an open-ended coaxial line: Part II - Experimental results IEEE Trans. Microw. Theory Tech. 30 87-92

Tubaro A, De Nunzio C, Trucchi A, Stoppacciaro A and Miano L 2008 The electromagnetic detection of prostatic cancer: evaluation of diagnostic accuracy Urology 72 340-4

Vander Vorst A, Rosen A and Kotsuka Y 2006 RF/microwave interaction with biological tissues (New York, NY: Wiley)

Vannelli A, Battaglia L, Poiasina E and Leo E 2010 Diagnosis of rectal cancer by Tissue Resonance Interaction Method BMC Gastroenterol. 1045

Vedruccio C and Vedruccio C R 2011 Non invasive radiofrequency diagnostics of cancer. The Bioscanner Trimprob technology and clinical applications J. Phys. Conf. Ser. 329012038

Wolf M, Gulich R, Lunkenheimer P and Loidl A 2012 Relaxation dynamics of a protein solution investigated by dielectric spectroscopy Biochim. Biophys. Acta 1824 723-30

Yoo D S 2004 The dielectric properties of cancerous tissues in a nude mouse xenograft model Bioelectromagnetics 25 492-7

Zhang H, Arslan T and Flynn B 2013 A single antenna based microwave system for breast cancer detection: Experimental results 2013 Loughborough Antennas and Propagation Conference, LAPC 2013 (IEEE) pp $477-81$ 